\title{
¿DE QUÉ Y DE QUIÉNES NARRA LA GENTE CUANDO RELATA NARRACIONES DE EXPERIENCIA PERSONAL? UN ANÁLISIS VARIACIONISTA DEL ESPAÑOL HABLADO EN SANTIAGO DE CHILE*
}

\author{
Silvana Maricel Guerrero González ${ }^{* *}$
}

\section{Resumen}

En el marco del enfoque variacionista laboviano, en esta investigación se analizan los temas y los protagonistas en una muestra de 192 narraciones de experiencia personal del español hablado en Santiago de Chile. Específicamente, nos proponemos comprobar si existe variación entre los factores sociodemográficos -sexo, edad y grupo socioeconómico de los hablantes- y el uso de dichos recursos, desde el punto de vista cuantitativo y cualitativo de análisis. El análisis de los datos demuestra que existe variación sociolingüística en el empleo de los temas y los protagonistas de las narraciones especialmente en relación con el factor sexo.

Palabras clave: sociolingüística variacionista, variación discursiva, narraciones de experiencia personal, temas y protagonistas.

\section{WHAT AND WHO TELLS PEOPLE WHEN HE RECOUNTS PERSONAL EXPERIENCE NARRATIVES? VARIATIONIST ANALYSIS IN SPEAKERS FROM SANTIAGO, ChILE}

\begin{abstract}
Following the Labovian variationist approach, this research study analyzes the use of the themes and characters in a sample of 192 oral personal experience narratives in the Spanish spoken in Santiago, Chile. We aim at establishing the frequency of use of this variables elements and testing whether there exists variation among the sociodemographic factors -sex, age, and socioeconomic group- of the speakers and the use of the said resources, in terms of quantitative and qualitative analysis. Our data analysis shows that there is sociolinguistic variation in the use of the themes and characters in personal experience narratives, specifically, regarding the sex factor.
\end{abstract}

Keywords: variationist sociolinguistic, discourse variation, personal experience narratives, themes and characters.

Recibido: 12-12- $2014 \quad$ Aceptado: 14-01-2015

\footnotetext{
* Este trabajo es parte de la tesis de doctorado Variación discursiva en narraciones de experiencia personal en el español hablado en Santiago de Chile, realizado en la Pontificia Universidad Católica de Chile.

** Chilena. Doctora en Lingüística de la Universidad de Chile. Santiago, Chile. siguerrero@u.uchile.cl
} 


\section{Introducción}

El creciente interés por el estudio de la variación sintáctica y discursiva en español ha dado origen a numerosas publicaciones que se han aproximado al estudio de variables lingüísticas a nivel de la sintaxis y el discurso, utilizando para ello los constructos teórico-metodológicos sugeridos por la sociolingüística laboviana (1983[1972]). Algunos aspectos de la variación sintáctica y discursiva en el habla de Santiago de Chile han sido estudiados por Prieto (1995-1996), Poblete (1998), SilvaCorvalán (2001), Prieto y San Martín (2002-2003), San Martín (2004-2005, 2011, 2013), Rojas, Rubio, San Martín y Guerrero (2012) y San Martín y Guerrero (2013). De manera específica, la narración de experiencia personal, desde el punto de vista laboviano, en el ámbito hispánico, ha sido estudiada, entre otros, por Silva-Corvalán (1987), Reyes (1996 y 2002), Shiro (2003), Soler (2004), Camargo (2004) y Guerrero (2011a, 2011b). No obstante lo anterior, estas investigaciones se han basado en el análisis cuantitativo de los datos, por lo que comenzar a hacer el vínculo entre lo cuantitativo y lo cualitativo se vuelve fundamental. Basándonos en la hipótesis de que existe variación sociolingüística, nos proponemos analizar los temas y los protagonistas de una muestra de 192 narraciones de experiencia personal de hablantes de Santiago de Chile en correlación con sus factores sociodemográficos-sexo, edad y grupo socioeconómico-. Para esto, propondremos una metodología específica que nos permita cuantificar, correlacionar y cualificar los datos que se desprendan de dichas narraciones.

El marco de nuestra la investigación -como queda de manifiesto- es la sociolingüística, en particular, desde el enfoque variacionista, pero con un fuerte componente cualitativo para el análisis de los temas de las narraciones. Asimismo, es esencial que se incluyan variables externas más allá del sexo, que es la que suele emplearse en estudios de esta naturaleza. En consecuencia, la investigación aquí proyectada se perfila como una contribución a la descripción sociolingüística del español hablado en Santiago de Chile, en particular, y al de la lengua española, en general. 


\section{Marco Teórico}

\section{La narración de experiencia personal}

La bibliografía sobre narraciones es extensa; sin embargo, el trabajo seminal de Labov y Waletzky (1967), así como el de Labov (1972), han demostrado ser por mucho el modelo de más alto rendimiento empírico en la investigación sobre el problema. En estricto sentido, Labov (1972: 360) define la narración como "one method of recapitulating past experience by matching a verbal sequence of clauses to the sequence of events which (it is inferred) actually occurred [un método de recapitulación de la experiencia pasada adecuando una secuencia verbal de proposiciones a la secuencia de sucesos que (se supone) ocurrieron realmente (la traducción es nuestra)]". De esta forma, la narración es considerada como un texto que expresa una serie de eventos que ocurren en el tiempo, pero donde, a su vez, se percibe de manera subyacente una relación causal o temática.

Por otra parte, el relato de experiencia personal es, metodológicamente, muy útil tanto en la propuesta laboviana como en nuestra investigación, pues es en la narración donde se obtendría el discurso vernáculo1 del sujeto, esto es, el tipo de habla que usaría cuando no está siendo sistemáticamente observado.

\subsection{Los temas y los protagonistas de las narraciones}

Las narraciones que conforman la muestra de nuestro estudio forman parte de la sección de narración de experiencias personales de la entrevista semidirigida hecha a 192 hablantes santiaguinos. Se trata, en concreto, de la narración mejor desarrollada por cada informante (cf. 3.1). Si bien estamos aludiendo de un solo tipo de narración -la de experiencia personal- los temas generales son variados, a saber, anécdotas, recuerdos, vergüenzas, sustos, entre otros que pudieran surgir en el marco de la entrevista. A partir de los temas mencionados, propuestos por los entrevistadores, los informantes elaboran y comparten sus historias, algunas con un mayor grado de involucramiento y otras que no necesariamente corresponden a relatos "dignos de contarse" en el sentido laboviano. Nosotros hemos optado por seleccionar aquellas que, por su alto nivel de involucramiento, son las mejores desarrolladas

1 Citando a Labov, Moreno Fernández (2012: pp. 181-182) señala que: “El vernáculo, en el que se presta al discurso propio una atención mínima, aporta los datos más sistemáticos por el análisis lingüístico. El vernáculo se define como la forma de hablar adquirida durante la preadolescencia". 
y, por lo mismo, pueden compararse. Dentro de los relatos, tanto los subtemas como sus personajes cobran vital importancia en un análisis variacionista. En relación con los temas, lo que se pretende en este estudio es clasificar cada narración en función de la temática que aborde, por ejemplo, asaltos, enfermedades, moda, viajes, vacaciones, entre muchos otros que se desprendan del análisis. A este respecto, como sabemos, son los estudios vinculados con la variable sexo, los que han dado cuenta de diferencias de género en las temáticas de las historias. Así, encontramos trabajos como los de Tannen (1990a, 1990b, 1996), Soler (2004) y Coates (2009), entre otros que consideran esta variable. Sin embargo, las variables edad y grupo socioeconómico no han tenido la misma relevancia en las investigaciones. Por consiguiente, los hallazgos que aquí se realicen constituirán un aporte al análisis sociolingüístico del discurso.

Junto con lo anterior, los personajes que llevan a cabo las acciones de las narraciones también podrían ser reflejo de diferencias que se pueden registrar entre los informantes santiaguinos. Adam y Lorda (1999, p. 156) postulan que "que el narrador y los personajes sean entes claramente diferenciados del autor no significa que no lleven en ellos "huellas" o tomen "préstamos" [...]. Los escritores se refieren en ocasiones a la naturaleza de la relación entre sus propias vidas y las vidas que crean en la ficción". Así, el narrador imagina y crea a sus personajes objetiva y subjetivamente. En este sentido, siguiendo el método inicial de Soler (2004), interesa, sobre todo, establecer si los protagonistas actúan solos o con otras personas, y quienes son, finalmente, los protagonistas de los relatos2. Así, nos proponemos examinar si el protagonista es la primera persona, es decir, el mismo narrador; si se trata más bien de terceras personas que no son el narrador; o si se trata de la primera y terceras personas en conjunto, es decir, del narrador de los hechos acompañado de otros personajes. En relación con la inscripción de la primera persona en la narración (yo), Calsamiglia y Tusón (1999, pp. 138-139) señalan que la persona que habla no es un ente abstracto, sino un sujeto social que se presenta ante los demás de una manera determinada. A medida que se construye el discurso también se construye el sujeto discursivo, quien se adapta a la situación específica de la comunicación modulando

2 Recordemos que en la investigación empírica de Soler (2004), el énfasis está puesto en determinar la existencia de terceras personas, y de haberlas, si se trata de hombres o mujeres. En nuestro caso, por tratarse de un análisis variacionista que incluye las variables independientes sexo, edad y grupo socioeconómico, el objetivo es más general, esto es, determinar de quién es el protagonismo en las narraciones. 
su posición a lo largo del discurso e intentando que su interlocutor lo reconozca de una manera y no de otra. A nuestro juicio, esto mismo se aplica a las terceras personas presentes en la historia contada, pues es el narrador (yo) quien construye a sus personajes y los presenta de una manera determinada ante el entrevistador.

Si bien estamos conscientes de que se trata de un análisis más bien general, por lo que implica encasillar en un único subtema toda la narración o de determinar quién es el real protagonista de los hechos principales, en especial, cuando hay relatos adosados a la historia principal, consideramos que una pesquisa de esta envergadura podría dar luces sobre posibles aspectos que podrían incorporarse en estudios cuyo foco sea la variación discursiva, sobre todo, asumiendo que los métodos desarrollados a este respecto son escasos.

\subsection{La variación según variables sociodemográficas: sexo, edad y grupo socioeconómico}

El desarrollo de la sociolingüística se basa en que gran parte de los hechos de variación lingüística varían en forma sistemática, si se los correlaciona con variables sociodemográficas como el grupo socioeconómico, la edad y el sexo de los informantes. Esto implica el hecho de que las variables lingüísticas estén en covariación sistemática con las variables permite a los individuos instalarse en un determinado espacio del espectro social (Coates, 2009).

Moreno Fernández (1998) señala que la investigación sociolingüística ha demostrado que las variables sociales que influyen sobre la variación lingüística lo hacen de una forma específica en cada comunidad de habla y respecto de fenómenos lingüísticos concretos. En este orden, Blas Arroyo (2005) también pone de relieve que no es posible reconocer de antemano qué tipo de variables sociales van a actuar sobre la variación lingüística, primero, porque la variabilidad no tiene por qué manifestarse de la misma forma en comunidades de habla diferentes $\mathrm{y}$, segundo, porque estos mismos factores no se configuran de la misma forma en sociedades distintas. Como ya señalamos, el factor sexo, el factor edad y el factor grupo socioeconómico serán las variables independientes empleadas en esta investigación sobre el carácter que asume la variación lingüística correspondiente al empleo de temas y de protagonistas de las narraciones, en una muestra del español hablado en Santiago de Chile. 


\section{Metodología}

\subsection{Corpus}

El corpus que sirve de base para nuestro estudio corresponde a 192 narraciones de experiencia personal extraídas de entrevistas sociolingüísticas pertenecientes al corpus del grupo de Estudio Sociolingüístico del Español de Chile $(\mathrm{ESECH})^{3}$, realizadas por los estudiantes en la cátedra de Sociolingüística, a cargo del Profesor Abelardo San Martín, de las carreras de Licenciatura en Lengua y Literatura Hispánicas y Lengua y Literatura Inglesas de la Universidad de Chile. Con base en el marco de la metodología variacionista propuesta por Labov, las entrevistas fueron realizadas a hombres y mujeres santiaguinos ${ }^{4}$ con características sociodemográficas congruentes.

En la situación de entrevista, los entrevistadores debían tratar de superar la paradoja del observador ${ }^{5}$ consiguiendo, de esta forma, una muestra significativa de discurso natural grabado (estilo vernáculo) de hablantes representativos de la comunidad de habla en estudio. El cuestionario utilizado para la realización de las mencionadas entrevistas consta de una sección cuya función es elicitar las narraciones de experiencia personal que conforman nuestro corpus. En relación con esto, es necesario precisar que se seleccionó la narración mejor desarrollada por cada informante, que es, básicamente, aquella que contiene la mayor cantidad de cláusulas narrativas en términos labovianos; de esta forma, se puede medir el mejor desempeño de cada uno de ellos y, posteriormente, compararlos.

El criterio de selección de la narración mejor desarrollada nos permitió controlar algunas variables intervinientes, como el cansancio y el desinterés de los sujetos considerando la duración de la entrevista.

3 El ESECH, grupo del cual formamos parte, está a cargo del Profesor del Departamento de Lingüística de la Universidad de Chile, Abelardo San Martín, a quien agradecemos la facilitación del corpus de esta investigación. El corpus del ESECH está conformado por entrevistas realizadas entre los años 2005 y 2012.

4 Los criterios de asignación de hablante nativo de Santiago de Chile siguen la propuesta de Prieto (1995-1996, pp. 399).

5 Según Labov (1983 [1972]), el objetivo de la investigación lingüística de una comunidad de habla consiste en estudiar la forma en cómo habla la gente cuando no está siendo sistemáticamente observada; sin embargo, sólo podemos obtener tales datos mediante la observación sistemática. A esto es a lo que se le ha denominado paradoja del observador. Este dilema se soluciona encontrando la manera de completar la entrevista formal con otros datos, o cambiar la estructura de la situación de la entrevista de una u otra manera. 
De este modo, se trata de narraciones que presentan un alto grado de involucramiento.

\subsection{Población y muestra}

En nuestra investigación se consideró la población constituida por hombres y mujeres de la Región Metropolitana de más de 20 años de edad. En lo referido a los tres grupos etarios que hemos considerado apelamos a tres momentos vitales (Blas Arroyo, 2005). Para la estratificación de los sujetos que conforman la muestra del estudio, se empleó el sistema de adscripción de estatus social empleada en ESECH que parte de una asignación de puntaje según las siguientes tres variables: 1) nivel educacional, 2) profesión u ocupación y 3) comuna de residencia. A cada una de estas variables se le otorgó una ponderación distinta, a saber: 3 puntos para el nivel educacional, 2 para la variable profesión u ocupación y 1 punto para la variable comuna de residencia. La escala incluye cuatro estratos socioeconómicos, a saber: bajo, medio bajo, medio y medio alto, para cuya diferencia se cauteló la consistencia entre las variables antes mencionadas de manera que respondieran a lo que Prieto (1995-1996), siguiendo a Lenski (1954), denominara congruencia de status.

El cuestionario se aplicó a una muestra del tipo "muestra por cuotas con afijación uniforme" (López Morales, 1994, p. 58). La muestra así conformada comprende un total de 192 entrevistas realizadas a igual número de sujetos, distribuidos como se indica en la Tabla 1.

Tabla 1. Proporción de sujetos según características sociodemográficas de sexo, edad y grupo socioeconómico

\begin{tabular}{l|l|l|l|l|l|l|l} 
& \multicolumn{2}{|c|}{$20-34$} & \multicolumn{3}{c|}{$35-54$} & \multicolumn{3}{c|}{55 y más } & \\
& H & M & H & M & H & M & Total \\
\hline Medio alto & 8 & 8 & 8 & 8 & 8 & 8 & $=48$ \\
\hline Medio & 8 & 8 & 8 & 8 & 8 & 8 & $=48$ \\
\hline Medio bajo & 8 & 8 & 8 & 8 & 8 & 8 & $=48$ \\
\hline Bajo & 8 & 8 & 8 & 8 & 8 & 8 & $=48$ \\
\hline & 32 & 32 & 32 & 32 & 32 & 32 & $=192$ \\
\hline
\end{tabular}




\section{Presentación, análisis y discusión de los resultados ${ }^{6}$}

Antes de dar paso a la exposición de los apartados mencionados, daremos cuenta de algunos aspectos generales del análisis. En primer término, presentaremos la clasificación de áreas temáticas derivadas de la muestra. Dichas áreas fueron derivadas a partir de la creación de una macroproposición de cada narración, la que, en algunos casos, coincidió con el resumen del relato que el mismo informante realizaba al momento de comenzar a contar su historia. Así, llegamos a determinar 12 categorías temáticas, que presentamos a continuación 7 .

1. Vergüenzas: hecho en el que el narrador sufre alguna vergüenza pública.

2. Hechos delictuales: es un hecho delictual del que el narrador (solo o acompañado) es víctima.

3. Accidentes: hecho accidental en el que el narrador se ve envuelto en una situación compleja en la que puede, incluso, resultar dañado.

4. Bromas y travesuras: situaciones ingeniosas que el narrador realiza o es testigo.

5. Hijos: es un hecho en el que el hijo (a) del narrador es el protagonista.

6. Peleas y golpizas: peleas en el que el narrador se ve envuelto.

7. Sueños premonitorios: es un hecho en el que el narrador relata su sueño premonitorio.

8. Sustos no paranormales: situaciones en las que el narrador (solo o acompañado) sufre situaciones no sobrenaturales que lo asustan, distintas de 5 .

6 Los ejemplos correspondientes solo serán expuestos, en ortografía convencional, en 4.4., a fin de no ser redundantes en relación con las secciones anteriores de este análisis. Cada ejemplo se transcribirá en ortografía convencional. Los nombres propios serán reemplazados por una letra en mayúscula y se identificarán las partes de la narración laboviana a través de un sistema de etiquetas del tipo $<>$.

7 Soler (2004, p. 101), basándose en Bublitz (1988, p. 23), sostiene que una forma de definir los temas es, precisamente, haciendo una abstracción que permita llegar a una palabra o a una categoría que refiera al contenido global de un tema, por lo tanto, la descripción de un tema puede realizarse a través del empleo de una proposición, un único nombre o una frase nominal compleja. Nosotros, como ya lo presentamos, hemos optado construir una macroproposición para determinar los ejes temáticos antes que abstracciones, que podrían resultar mucho más subjetivas. 
9. Sustos paranormales: situaciones en las que el narrador (solo o acompañado) sufre situaciones que lo asustan y que se asocian a fenómenos paranormales.

10. Vacaciones y aventuras: hecho en que el narrador cuenta sus aventuras vividas en el marco de sus vacaciones.

11. Otros: recuerdos de situaciones agradables no incluidos en las categorías anteriores.

12. Otros: recuerdos de situaciones desagradables no incluidos en las categorías anteriores.

Con la descripción temática antes expuesta, hemos querido mostrar que se trata de categorías que, efectivamente, se diferencian unas de otras. Lo esencial en todos los casos es la síntesis que puede hacerse de la historia.

\subsection{Análisis de los efectos del factor sexo en la construcción de narraciones de experiencia personal}

\subsubsection{Análisis de los temas de las narraciones}

Tal como señaláramos anteriormente, si bien estamos aludiendo de un solo tipo de narración -la de experiencia personal- los temas generales tratados en nuestras historias son variados, esto es, anécdotas, recuerdos, vergüenzas y sustos. No obstante, a partir de estos temas, los informantes tienen la posibilidad de elaborar y compartir otros más específicos. En lo que sigue presentaremos la nómina de temáticas que relevamos en esta investigación y analizaremos su comportamiento en relación con el factor sexo.

Tabla 2. Temas de las narraciones según el factor sexo

\begin{tabular}{l|l|l|l}
\hline Categorías temáticas & Frecuencia Hombres & Frecuencia Mujeres & Totales \\
\hline Vergüenzas & 19 & 28 & 47 \\
\hline Hechos delictuales & 20 & 12 & 32 \\
\hline Accidentes & 14 & 8 & 22 \\
\hline Hijos & 1 & 15 & 16 \\
\hline $\begin{array}{l}\text { Sustos no } \\
\text { sobrenaturales }\end{array}$ & 9 & 7 & 16 \\
\hline
\end{tabular}




\begin{tabular}{|c|c|c|c|}
\hline $\begin{array}{l}\text { Otros recuerdos } \\
\text { desagradables }\end{array}$ & 5 & 9 & 14 \\
\hline Bromas y travesuras & 8 & 4 & 12 \\
\hline $\begin{array}{l}\text { Otros recuerdos } \\
\text { agradables }\end{array}$ & 6 & 5 & 11 \\
\hline Peleas y golpizas & 4 & 2 & 6 \\
\hline Sueños premonitorios & 5 & 1 & 6 \\
\hline Sustos sobrenaturales & 4 & 1 & 5 \\
\hline Vacaciones y aventuras & 1 & 4 & 5 \\
\hline
\end{tabular}

Como se puede apreciar en la tabla recién expuesta, en este estudio relevamos 12 ejes temáticos en las 192 narraciones que constituyen nuestra muestra de investigación. De dichos ejes, no hay ningún tema que no sea cubierto por alguna de las 96 narraciones de hombres y de mujeres. Este hallazgo se asemeja, en parte, a aquellos encontrados, por ejemplo, por Soler (2004), quien en su estudio sobre relatos de hombres y mujeres de Bogotá (Colombia), concluye que los hombres abarcan una mayor variedad de temas. Nosotros no encontramos más variedad de temas, pero sí que los hombres usan con más frecuencia la mayoría de ellos, es lo que ocurre, por ejemplo, con los hechos delictuales, los accidentes, los sustos no sobrenaturales, las bromas o travesuras, otros recuerdos agradables, las peleas y golpizas, los sueños premonitorios y los sustos sobrenaturales. Además, Soler (2004) observa que los informantes de ambos sexos coinciden en cuanto a su frecuencia en los temas más tratados en las historias. Nosotros observamos que los tres temas con mayor frecuencia en nuestras narraciones presentan una diferencia de frecuencia que va, por lo menos, desde los seis casos entre hombres y mujeres.

Al comparar las temáticas seleccionadas por hombres y mujeres para ser narradas con mayor exactitud ${ }^{8}$, hay tres aspectos interesantes de destacar, pese a que las mujeres no presentan mayor variedad temática que los hombres, como han sugeridos otros estudios que abordan este aspecto. En primera instancia, que los temas que ellas desarrollan y que no son los seleccionados por los hombres que integran la muestra, son los que suelen presentarse en la literatura como más característicos del sexo

8 Recordemos que estamos trabajando con la narración mejor desarrollada por cada sujeto. 
femenino (cf. Tannen, 1996; Prieto y San Martín, 2002-2003; Soler, 2004 y Coates, 2009), a saber, hijos y vacaciones y aventuras. Luego, es prudente destacar el caso del eje que involucra a hijos, porque si bien hay una narración de un hombre que lo incluye, son 15 los relatos de mujeres que abordan este tema, lo que implica una importante diferencia ${ }^{9}$. Asimismo, hay temas que se perfilan como más típicos de hombres, como los hechos delictuales, donde además suelen ser héroes. Lo mismo ocurre con los accidentes, las bromas y travesuras y las peleas y golpizas. En tercer lugar, queremos poner de relieve que, aunque observamos estas diferencias, tanto hombres como mujeres se comportan de manera muy similar en la selección de sus áreas temáticas en las narraciones que conforman nuestra muestra de estudio.

\subsubsection{Análisis de los protagonistas de las narraciones}

En esta sección presentaremos el detalle de la variación entre el protagonista (o los protagonistas) de los sucesos y la variable externa sexo. En la Tabla 3 podemos observar lo que ocurre entre la selección de los protagonistas de la narración y dicho factor externo.

Tabla 3. Protagonistas de las narraciones según el factor sexo

\begin{tabular}{l|l|l|l}
\multicolumn{1}{c|}{ Protagonistas } & \multicolumn{1}{c|}{ Frecuencia Hombres } & Frecuencia Mujeres & Totales \\
\hline Primera persona & 52 & 34 & 86 \\
\hline $\begin{array}{l}\text { Primera y tercera } \\
\text { persona }\end{array}$ & 40 & 47 & 87 \\
\hline Tercera persona & 4 & 15 & 19 \\
\hline
\end{tabular}

Es interesante la tabla precedente, porque, como es evidente, son los hombres quienes tienden a ser los protagonistas de sus propias historias, esto es, un 60,5\% (52/86), mientras que las mujeres tienen esta tendencia en muchos menos casos, a saber, en un 39,5\% (34/86). Esta importante presencia del yo como protagonista de los relatos de hombres es un hallazgo que se repite en Soler (2004) en su estudio con hombres y mujeres de Bogotá. En este orden, son ellas las que suelen incluirse con terceras personas en roles protagónicos, en concreto en un 54\% (47/87) de las

9 En relación con esto, Prieto y San Martín (2002-2003) ponen de relieve los factores que Coates (1989) considera que podrían explicar ciertos rasgos del habla femenina. Entre ellos se halla el tópico, que, según Coates, alude a los roles de la mujer de esposa, novia y madre. 
narraciones y las que incluyen casi cuatro veces más que los hombres solo a terceras personas como protagonistas, a saber, en 15/19 (78,9\%) ocasiones.

Junto con el reporte anterior, hemos efectuado una prueba ji cuadrado $^{10}$ a fin de determinar la asociación de la variable sexo con el uso de personas en los relatos. La prueba mencionada señala que el factor sexo es estadísticamente significativo en el empleo de primeras personas en las narraciones, donde el valor de $\mathrm{p}=0,009$, y en el uso de terceras personas como protagonistas, en cuyo caso el valor de $p=0,008$. En consecuencia, el empleo mayoritario de primeras personas como actores principales de los sucesos narrados parece ser una característica propia de los hombres, en tanto que la preferencia por incluir a otros sujetos como protagonistas sería una tendencia más propia de las mujeres que conforman la muestra de estudio.

\subsubsection{Síntesis, discusión y proyección de resultados}

A modo de síntesis de este primer apartado, queremos destacar, en primer lugar, que en lo que respecta a los temas de las narraciones, pese a que no se observó una mayor variedad en las narraciones de mujeres, hay temas que parecen ser casi exclusivos de sus relatos, como aquel que involucra a los hijos y el que habla de vacaciones y aventuras. También resultó ser interesante el hecho de estos temas que desarrollan, de manera casi exclusiva, las informantes femeninas, son los que suelen presentarse en la literatura como más característicos de dicho sexo. A este respecto, es necesario mencionar que tal como señalan autoras como Tannen (1996), Soler (2004) y Coates (2009), entre otras que han abordado estudios de este tipo, las mujeres tienden a introducir nuevos temas en los relatos, a diferencia de los hombres, que suelen recurrir a los mismos, lo que hemos corroborado en nuestra investigación, pues son ellas quienes narran sobre sueños premonitorios, vacaciones y aventuras y recuerdos desagradables en los relatos de la nuestra muestra. Así, es relevante considerar que si bien las primeras investigaciones en este ámbito tuvieron un enfoque meramente descriptico, las siguientes se situaron en la esfera explicativa (o interpretativa). En este último caso, los dos enfoques predominantes

10 Utilizamos el procesador SPSS.15 para las pruebas estadísticas. La significación se alcanzaba con $\mathrm{p}<0,05$. La elección de esta prueba, a diferencia de lo que ocurre con el análisis de los temas, queda plenamente justificada porque los datos permiten realizarla. 
son el de la dominación y el de las diferencias culturales. De acuerdo con Prieto y San Martín:

El primero interpreta las diferencias lingüísticas como consecuencia del desequilibrio de poder que, desde tiempos inmemoriales, ha existido en detrimento de la mujer; el segundo enfoque, en cambio, asienta el énfasis explicativo en el supuesto fundamental de que los hombres y las mujeres hablan de modo diferente, o actúan de modo diferente en la interacción conversacional, debido a que su socialización transcurre en diferentes subculturas sociolingüísticas. (Prieto y San Martín, 2002-2003, pp. 271-272)

Los hallazgos relevados en esta investigación parecen acercarse a las investigaciones cuyo foco es el de las diferencias culturales, aunque el enfoque de la dominación está igualmente presente cuando son los hijos el eje central de la narración. En consecuencia, nos parece pertinente considerar la posibilidad de realizar futuros trabajos centrados en la narrativa, pero con técnicas de recolección de datos como la entrevista en profundidad, ya que de esta manera podremos tener una mayor cantidad de datos que nos ayuden a comprender el rol de madre y la construcción de la identidad de madre en nuestra comunidad de habla. Así, valoramos propuestas de investigación como la de Gajardo (en preparación), cuyo objetivo principal es justamente analizar la construcción identitaria de madre/trabajadora en un grupo de madres de los niveles alto, medio y bajo de Santiago de Chile, tomando como herramientas teórico-analíticas las provenientes de los estudios críticos del discurso. En este orden, es igualmente prudente intentar determinar la incidencia que tienen en la actualidad los denominados enfoques de la dominación y de las diferencias culturales, como posibles explicaciones en el comportamiento variable en las narraciones de hombres y de mujeres (cf. Prieto y San Martín, 2002-2003), en especial, porque en la actualidad chilena, la lucha por la igualdad de género es cada vez mayor. Asimismo, habría que intentar averiguar por qué las historias de las mujeres y de los hombres difieren en la naturaleza del contexto y los temas, en especial, en el heroísmo y en el protagonismo presente en las narraciones masculinas (cf. Johstone, 1993 y Soler, 2003). La pregunta de fondo, en este caso, es si la mujer efectivamente habla más de sentimientos en tanto que el hombre se centra en las acciones, como lo propusiera Talbot (1998, citado en Soler 2004; Holmes, 1997, citado en Macaulay, 2003 y Johnstone, 1990). 
Nosotros hemos demostrado que existe una división de temas entre los sexos, pero el porqué de la elección de esos temas es lo que queda por averiguar.

Desde el campo de la psicología, estudios como el de Sharim (2005), postulan que las mujeres narran de manera "femenina", situando a la pareja en el centro de la historia y centrándose en el mundo de los afectos, del hogar y de la reproducción, mientras que los hombres se centran en el trabajo, en su formación profesional o su participación política, y el poder se refleja en su masculinidad y califican de "macho cumplidor"; señalan incluso que para entender su historia de pareja primero debe conocerse su propia historia. Lo femenino y lo masculino se ven, sin embargo, matizados por la constante lucha de poder en beneficio de la igualdad de género.

En lo que se refiere a los protagonistas de las narraciones, se percibió que son los hombres quienes tienen mayor tendencia a hablar de ellos mismos, situándose como actores principales de los acontecimientos que narran, y que son las mujeres quienes tienden a incluir a terceras personas con roles protagónicos, lo que, además, se comprobó mediante un análisis estadístico. En este contexto, no deja de ser relevante cómo los hombres se sitúan como héroes de las historias cuyo tema son los hechos delictuales, es decir, ellos, en su mayoría, logran resolver los problemas mientras que las mujeres se constituyen como víctimas (cf. 4.4.2.). Estos hallazgos coinciden con los de Johnstone (1993), quien concluye que los hombres cuentan relatos, por ejemplo, de pruebas en las que se ven enfrentados a otros hombres o a la naturaleza, de hazañas que exaltan sus habilidades, su coraje o su ingenio, y casi nunca incluyen a mujeres como protagonistas de sus narraciones. Los relatos de mujeres, por su parte, son sobre la sociedad y rara vez se presentan ellas mismas como protagonistas de la historia, papel que es asignado a hombres o a mujeres indistintamente. En el ámbito hispánico, Soler (2004) observa que los hombres, en general, hablan de ellos mismos o incluyen a otros hombres en los relatos, a diferencia de las mujeres que suelen hablar de otros, sean hombres o mujeres. Asimismo, apreció que las narraciones masculinas tienden a tener buenos resultados, en tanto que las femeninas, con mayor frecuencia, se resuelven con malos resultados. En nuestra investigación, como ya lo hemos precisado, este comportamiento es el habitual, por consiguiente, la mujer se transforma en la víctima y el hombre en el héroe de sus historias, lo que parece coincidir con el enfoque de las diferencias culturales más arriba descrito. 


\subsection{Análisis de los efectos del factor edad en la construcción de narraciones de experiencia personal}

\subsubsection{Análisis de los temas de las narraciones}

En este apartado expondremos la nómina de temáticas que relevamos en esta investigación y analizaremos su comportamiento en relación con el factor edad. La lista así constituida es la que se muestra en la Tabla 4, que presentamos a continuación.

Tabla 4. Temas de las narraciones según el factor edad

\begin{tabular}{l|l|l|l|l}
\hline Categorías Temáticas & \multicolumn{1}{|c|}{$\begin{array}{c}\text { Frecuencia } \\
20 \text { a } 34 \text { años }\end{array}$} & $\begin{array}{c}\text { Frecuencia } \\
35 \text { a } 54 \text { años }\end{array}$ & $\begin{array}{c}\text { Frecuencia } \\
55 \text { años y más }\end{array}$ & Totales \\
\hline Vergüenzas & 14 & 19 & 14 & 47 \\
\hline Hechos delictuales & 10 & 10 & 12 & 32 \\
\hline Accidentes & 6 & 8 & 8 & 22 \\
\hline Hijos & 3 & 7 & 6 & 16 \\
\hline $\begin{array}{l}\text { Sustos no } \\
\text { sobrenaturales }\end{array}$ & 6 & 6 & 4 & 16 \\
\hline $\begin{array}{l}\text { Otros recuerdos } \\
\text { desagradables }\end{array}$ & 3 & 4 & 7 & 14 \\
\hline Bromas y travesuras & 9 & 0 & 3 & 12 \\
\hline $\begin{array}{l}\text { Otros recuerdos } \\
\text { agradables }\end{array}$ & 4 & 3 & 4 & 11 \\
\hline Peleas y golpizas & 2 & 1 & 3 & 6 \\
\hline Sueños premonitorios & 3 & 1 & 2 & 6 \\
\hline Sustos sobrenaturales & 2 & 2 & 1 & 5 \\
\hline $\begin{array}{l}\text { Vacaciones y } \\
\text { aventuras }\end{array}$ & 2 & 3 & 0 & 5 \\
\hline
\end{tabular}

Tomando en consideración que tantoel factor edad comoel factor grupo socioeconómico no han sido objeto de estudio de una investigación de las características de la que presentamos, es interesante llamar la atención respecto de qué ocurre más allá del factor sexo que, como sabemos, ha sido ampliamente estudiado. En este sentido, en relación con el factor edad, observamos en los tres grupos la presencia de empleo de casi la totalidad de los ejes temáticos que relevamos. Asimismo, es interesante que todos los temas están distribuidos con frecuencias muy similares en los tres grupos etarios en estudio, aunque destacamos el caso de las 
vergüenzas, que son la temática más utilizada por los integrantes del grupo de edad intermedia; de las travesuras y bromas, mayoritariamente utilizadas por los jóvenes; los hijos, que también aparecen en el grupo de 35 y 54 años de manera preponderante; y otros recuerdos desagradables, que son más frecuentes en la gente del tercer grupo etario. Estos cuatro ejes temáticos son los que muestran mayor variedad en su distribución. A este respecto, Coupland, Coupland y Giles (1991, citados por Valles, 2006, p. 886) señalan que "la conversación entre adultos mayores y personas jóvenes contiene estrategias discursivas que reflejan y refuerzan creencias y estereotipos relacionados con la edad y con el proceso de envejecer", por lo que, en especial, los relatos sobre travesuras y bromas y aquellos que hablan de otros recuerdos desagradables podrían vincularse con estereotipos muy vinculados con la edad de los informantes.

\subsubsection{Análisis de los protagonistas de las narraciones}

También es relevante que analicemos el comportamiento de la inclusión de primera y terceras personas, o de ambas, como protagonistas de los relatos que conforman nuestra muestra de estudio en relación con el factor edad. Dicho comportamiento es el que se expone en la tabla que sigue.

Tabla 5. Protagonistas de las narraciones según el factor edad

\begin{tabular}{l|l|l|l|l}
\multicolumn{1}{c|}{ Protagonistas } & \multicolumn{1}{|c|}{$\begin{array}{c}\text { Frecuencia } \\
20 \text { a 34 años }\end{array}$} & $\begin{array}{c}\text { Frecuencia } \\
35 \text { a 54 años }\end{array}$ & $\begin{array}{c}\text { Frecuencia } \\
55 \text { años y más }\end{array}$ & Totales \\
\hline Primera persona & 26 & 27 & 33 & 86 \\
\hline $\begin{array}{l}\text { Primera y tercera } \\
\text { persona }\end{array}$ & 32 & 30 & 25 & 87 \\
\hline Tercera persona & 6 & 7 & 6 & 19 \\
\hline
\end{tabular}

Lo interesante de la tabla precedente es que los tres grupos de edad en estudio se comportan de manera muy similar en la elección de sus protagonistas, por lo tanto, a diferencia de lo ocurrido con el factor sexo, en este caso la variación es mínima, esto es, un máximo de siete casos de diferencia en sus frecuencias, lo que, considerando que son 64 narraciones por grupo de edad y que en muy pocos casos se emplea la tercera persona de manera exclusiva, es mínimo. 
A fin de demostrar de manera empírica nuestras observaciones anteriores, hemos desarrollado un análisis estadístico utilizando la prueba de ji cuadrado. Dicho análisis da cuenta de que ninguna de las tres formas variables resulta estadísticamente significativa cuando se las correlaciona con el factor edad.

\subsubsection{Síntesis, discusión y proyección de resultados}

A modo de síntesis, queremos destacar, en primer lugar, que en lo que respecta a los temas y a los protagonistas de las narraciones, no se percibió gran variedad. Sin embargo, es interesante que respecto de las temáticas abordadas, haya algunas como las vergüenzas, las travesuras o bromas, los hijos y otros recuerdos desagradables que sí parecen estar más marcadas en su uso. Así, las travesuras y bromas, por ejemplo, suelen aparecer en el grupo joven de la muestra (20 a 34 años) y transcurran en el colegio o en la universidad, probablemente porque son recuerdos del pasado más reciente de los informantes. De igual forma, los temas asociados con otros recuerdos desagradables se ubican sobre todo en el grupo de 55 años y más, como si se tratara de un tema más próximo a los hablantes de este grupo etario. A este respecto, los estudios a nivel de discurso y, en particular, sobre interacción discursiva, serían fundamentales para profundizar en aspectos como el descrito.

En otro orden, respecto de la elección de los protagonistas de los relatos, como ha quedado demostrado, este factor externo no mostró variación, por lo que concluimos que sigue siendo el factor sexo el más pertinente para realizar análisis de este tipo. De todas formas, es relevante haber propuesto una investigación que incluya datos empíricos que comprueben que es, quizás, el factor sexo, el único que realmente condiciona la variación temática y de protagonistas de las narraciones de experiencia personal.

\subsection{Análisis de los efectos del factor grupo socioeconómico en la construcción de narraciones de experiencia personal}

\subsubsection{Análisis de los temas de las narraciones}

Como ha quedado claro en los dos apartados anteriores, es menester desarrollar un análisis que nos permita determinar si es que hay variación o alguna tendencia en relación con los temas de las narraciones y el factor 
grupo socioeconómico, sobre todo, porque esta variable externa ha sido muy poco considerada en estudios de esta naturaleza y, de hecho, no existen investigaciones ni en Chile ni en el ámbito hispánico, en general, que aludan a ella en los términos que aquí proponemos. La distribución temática según el factor grupo socioeconómico es precisamente lo que muestra la Tabla 6.

Tabla 6. Temas de las narraciones según el factor grupo socioeconómico

\begin{tabular}{l|l|l|l|l|l}
\hline Categorías Temáticas & $\begin{array}{l}\text { Frecuencia } \\
\text { Grupo MA }\end{array}$ & $\begin{array}{c}\text { Frecuencia } \\
\text { Grupo M }\end{array}$ & $\begin{array}{l}\text { Frecuencia } \\
\text { Grupo MB }\end{array}$ & $\begin{array}{c}\text { Frecuencia } \\
\text { Grupo B }\end{array}$ & Totales \\
\hline Vergüenzas & 11 & 18 & 6 & 12 & 47 \\
\hline Hechos delictuales & 8 & 9 & 9 & 6 & 32 \\
\hline Accidentes & 4 & 3 & 9 & 6 & 22 \\
\hline Hijos & 1 & 3 & 3 & 9 & 16 \\
\hline $\begin{array}{l}\text { Sustos no } \\
\text { sobrenaturales }\end{array}$ & 3 & 7 & 4 & 2 & 16 \\
\hline $\begin{array}{l}\text { Otros recuerdos } \\
\text { desagradables }\end{array}$ & 6 & 1 & 3 & 2 & 14 \\
\hline Bromas y travesuras & 6 & 0 & 6 & 0 & 12 \\
\hline $\begin{array}{l}\text { Otros recuerdos } \\
\text { agradables }\end{array}$ & 3 & 1 & 4 & 3 & 11 \\
\hline Peleas y golpizas & 1 & 1 & 0 & 4 & 6 \\
\hline Sueños premonitorios & 3 & 1 & 1 & 1 & 6 \\
\hline Sustos sobrenaturales & 1 & 1 & 2 & 1 & 5 \\
\hline Vacaciones y aventuras & 1 & 1 & 1 & 2 & 5 \\
\hline
\end{tabular}

A partir de la Tabla 6, podemos destacar que, en general, los temas se presentan de manera bastante homogénea en los cuatro grupos socioeconómicos en estudio (medio alto, medio, medio bajo y bajo); de hecho, son los temas que hablan de vergüenzas, los que involucran hijos (el segundo más frecuente en el bajo), los que hablan de otros recuerdos desagradables y los que aluden a travesuras y bromas los que, podríamos decir, presentan mayor variabilidad en su distribución. En relación con el tema que involucra hijos, nos parece especialmente relevante la alta frecuencia con que lo desarrollan las mujeres pertenecientes al grupo socioeconómico bajo de la escala social. En un ejercicio donde enumeramos los cinco temas más frecuentes por grupo socioeconómico, solo en el grupo bajo las narraciones centradas en los hijos son destacables. En ningún caso estamos hablando de significatividad, pero sí es interesante que sea el segundo tema más utilizado en este grupo social. 
Por otro lado, debemos precisar que de los cuatro grupos en estudio, es el medio alto el único que incluye en sus relatos todos los temas relevados, y los tres grupos restantes solo incluyan 11/12 ejes temáticos. Según lo esbozado en 4 . de la presentación ya análisis de los resultados, los datos, en este caso, son más bien de orden descriptivo, por lo que es necesario mirar con cautela los hallazgos aquí presentados, en especial, aquellos que se vinculan con la variación. Por consiguiente, ponemos el énfasis en la variación de distribución de temas en las narraciones según los grupos socioeconómicos, aunque de manera muy general.

\subsubsection{Análisis de los protagonistas de las narraciones}

Así como lo hemos analizado en relación con el factor sexo y con el factor edad, también es relevante que analicemos el comportamiento de la inclusión de primera y terceras personas, o de ambas, en nuestras narraciones en relación con el factor grupo socioeconómico. Dicha distribución es la que observamos en la tabla siguiente.

Tabla 7. Protagonistas de las narraciones según el factor grupo socioeconómico

\begin{tabular}{l|l|l|l|l|l}
\hline \multicolumn{1}{c|}{ Protagonista } & $\begin{array}{l}\text { Frecuencia } \\
\text { Grupo MA }\end{array}$ & $\begin{array}{c}\text { Frecuencia } \\
\text { Grupo M }\end{array}$ & $\begin{array}{l}\text { Frecuencia } \\
\text { Grupo MB }\end{array}$ & $\begin{array}{c}\text { Frecuencia } \\
\text { Grupo B }\end{array}$ & Totales \\
\hline Primera persona & 18 & 30 & 20 & 18 & 86 \\
\hline $\begin{array}{l}\text { Primera y tercera } \\
\text { persona }\end{array}$ & 27 & 15 & 22 & 23 & 87 \\
\hline Tercera persona & 3 & 3 & 6 & 7 & 19 \\
\hline
\end{tabular}

En este caso, a diferencia de lo ocurrido según el factor edad, observamos un poco más de variedad en la distribución de los datos. En lo que respecta a la inclusión de primera persona es el grupo medio el que supera ampliamente a los demás, con 30/86 (34,9\%) casos totales, en tanto que los grupos medio alto, medio bajo y bajo se comportan de manera muy similar. Por su parte, en la inclusión de primera y terceras personas son los informantes del grupo medio alto quienes más optan por esta estrategia $(27 / 87,31 \%$ ) y en el grupo medio disminuye abruptamente su frecuencia (15/87). Finalmente, aquellos relatos que solo incluyen tercera persona se presentan mayoritariamente en los grupos socioeconómicos medio bajo y bajo (6/19 y $7 / 19$ casos, respectivamente). 
Por su parte, la prueba ji cuadrado que hemos utilizado para determinar la correlación entre el factor grupo socioeconómico y las formas variables en estudio señala que solo el uso de primera persona como protagonista de las historias resulta estadísticamente significativo, pues el valor de $\mathrm{p}=0,039$, por lo tanto, podría tratarse de una constante el hecho de que en el grupo medio de la escala socioeconómica sea mayor la inclusión del narrador de los hechos como su protagonista.

\subsubsection{Síntesis, discusión y proyección de resultados}

A modo de síntesis del tercer apartado, queremos destacar que en lo que respecta a los temas de las narraciones, si bien solo en cuatro categorías hubo variabilidad en la distribución según los grupos socioeconómicos, esto es, las vergüenzas, los que involucran hijos, los que hablan de otros recuerdos desagradables y los que aluden a travesuras y bromas, es de gran relevancia que haya sido el grupo socioeconómico medio alto el único que presentó los 12 ejes temáticos en sus narraciones, ya que hipotéticamente pensamos que los grupos bajos, por acercarse más a comunidades con oralidad primaria, tendrían mayor variedad en sus temas elegidos, como proponen Tannen (1996), Prieto y San Martín (2002-2003) y Soler (2004). Por lo mismo, futuras investigaciones que consideren este factor social nos ayudarán a comprender mejor el vínculo que se ha tendido a establecer entre las culturas con oralidad primaria y las narraciones que producen, ¿no serían estas narraciones de experiencia personal, en el sentido laboviano?

Por otra parte, los hallazgos obtenidos en el análisis de los protagonistas de las narraciones solo nos permiten afirmar que el empleo de primera persona es menos característico en los extremos de la escala socioeconómica, lo que es muy interesante, porque esto podría repercutir, por ejemplo, en que los integrantes del grupo bajo de la escala social construyan relatos menos ricos en cuanto a recursos de evaluación. Así, podríamos pensar que al hablar menos de historias exclusivamente propias, los informantes se involucran menos $y$, en consecuencia, evalúan menos, pues no se apropian de los sucesos y hacen de su relato uno reportable, pero más plano y lineal. Se trata, por supuesto, de una hipótesis sujeta a comprobación. 


\subsection{Análisis de la interacción entre las variables independientes}

\subsubsection{Análisis de los temas de las narraciones}

De acuerdo con el análisis presentado en los tres apartados precedentes, en este tenemos como objetivo exponer una síntesis que nos permita vincular los hallazgos anteriores con las tres variables externas en estudio, sexo, edad y grupo socioeconómico, en interacción. En consecuencia, presentamos la Tabla 8, con los datos totales de los ejes temáticos relevados en esta investigación.

Tabla 8. Temas de las narraciones según los factores sexo, edad y grupo socioeconómico

\begin{tabular}{l|c|c}
\multicolumn{1}{c|}{ Categorías Temáticas } & Totales & Porcentaje de Frecuencia \\
\hline Vergüenzas & 47 & $24,5 \%$ \\
\hline Hechos delictuales & 32 & $16,7 \%$ \\
\hline Accidentes & 22 & $11,5 \%$ \\
\hline Hijos & 16 & $8,3 \%$ \\
\hline Sustos no sobrenaturales & 16 & $8,3 \%$ \\
\hline Otros recuerdos desagradables & 14 & $7,3 \%$ \\
\hline Bromas y travesuras & 12 & $6,3 \%$ \\
\hline Otros recuerdos agradables & 11 & $5,7 \%$ \\
\hline Peleas y golpizas & 6 & $3,1 \%$ \\
\hline Sueños premonitorios & 6 & $3,1 \%$ \\
\hline Sustos sobrenaturales & 5 & $2,6 \%$ \\
\hline Vacaciones y aventuras & 5 & $2,6 \%$ \\
\hline Total & $\mathbf{1 9 2}$ & $\mathbf{1 0 0} \%$ \\
\hline
\end{tabular}

Como se puede ver en la Tabla 8, el tema más productivo resultó ser el que se vincula con vergüenzas con $47 / 192(24,5 \%)$ casos totales. Le siguen, en orden de frecuencia, los hechos delictuales con 32/192 (16,7\%) casos y los accidentes con 22/192 (11,5\%) casos. Posteriormente, se hallan las narraciones que hablan de hijos y las que hablan sobre sustos no sobrenaturales con 16/192 (8,3\%) casos cada eje temático y, de ahí en adelante, las frecuencias siguen en orden descendente hasta llegar a los sustos sobrenaturales y a las vacaciones y aventuras con 5/192 narraciones, lo que equivale apenas al $2,6 \%$ del total de la muestra en cada categoría. 
En relación con las tres variables externas en estudio, podemos mencionar que solo la variable sexo mostró mayor variabilidad, ya que en los casos de la edad y los grupos socioeconómicos, las frecuencias se distribuyeron, en general, de manera muy homogénea, excepto en los casos que fueron explicitados en los apartados correspondientes. De todas formas, si pudiéramos poner de relieve algunos casos, es conveniente destacar el hecho de que son las mujeres de 35 a 54 años del grupo socioeconómico bajo quienes tienden a incluir a sus hijos en las narraciones. En este sentido, los factores que menciona Coates (1989, citada en Prieto y San Martín, 2002-2003), como posibles causas de las características del habla femenina también se observan en el habla de Santiago de Chile, pero más específicamente en las mujeres de los grupos bajos de la escala social. Por otro lado, es destacable que los hombres, preferentemente, de edad joven (20 a 34 años) de los grupos medio alto y medio bajo son quienes tienen tendencia a narrar respecto de travesuras y bromas, todas muy vinculadas con su paso por la escuela o por la universidad.

Consideramos que los mencionados constituyen los hallazgos más interesantes al cruzar las variables independientes en estudio en relación con los temas que elaboran de mejor manera nuestros informantes.

A fin de clarificar la presencia de estos temas en las narraciones que conforman nuestra muestra de estudio, presentamos los ejemplos (1), (2) y (3).

El ejemplo (1) corresponde a una vergüenza, el tema con mayor frecuencia entre las 192 narraciones que conforman nuestra muestra de estudio.

(1) <orientación > una en el colegio / fue que mi hermana usó mis zapatillas y ella era de los pies ${ }^{11}$ y las dejó en la sala/ o sea/ las compartíamos porque era un tiempo como de crisis en mi casa/ compartí/ compartíamos con ella las zapatillas y ella los pies hediondos $<$ / orientación $>$ entonces las zapatillas al final de la mis zapatillas las dejó al final de la sala <acción de complicación> y cuando llegó la hora del examen no las usé porque me dio vergüenza / todo el mundo se había dado cuento que estaban ahí que estaban hediondas así que no las usé </acción de complicación $><$ resolución $>$ incluso las dejé ahí </ resolución $>$. (BIIM030)

11 La informante se toca la nariz haciendo alusión a pies hediondos. 
En el ejemplo precedente observamos cómo la informante describe una de sus mayores vergüenzas. En términos de estructura, se presencia una narración bastante completa, excepto por la falta de resumen y de coda, al igual que en el ejemplo (2), elementos que, no obstante, son muy prescindibles en un relato. En ambos casos, además, vemos como el empleo de primera persona como protagonista de los sucesos se vuelve regular, lo que va a ocurrir, sobre todo, en las narraciones de informantes hombres de la muestra en estudio. En este ejemplo, en particular, si bien la narradora presenta a otros personajes en el marco de los acontecimientos narrados, es "su" vergüenza lo esencial en la historia; todo lo anterior funciona como orientación de los hechos.

El segundo ejemplo que presentamos corresponde a un hecho delictual.

(2) <orientación> un día/ aprovechando horario de colación fui a hacer compras para no tener que pasar en la tarde $<$ / orientación> <acción de complicación > y una persona cruzó/ y yo iba con las manos ocupadas con los bolsos / y me sacó el bolsillo completo </acción de complicación > afortunadamente ya no tenía plata/ así que solamente los documentos $<$ resolución> así que le grité que me devolviera los documentos y él los tiró al suelo al ver que eran puro papel y recuperé los documentos y también recuperé el bolsillo de la camisa $<$ / resolución>. (MIIIH131)

Como ya señalamos, otro de los temas que mostró una de las mayores frecuencias en nuestras narraciones es el que corresponde a hechos delictuales. En este caso, se narra la historia de un robo, cuyo fin beneficia al informante, porque, a pesar del mal momento, recupera tanto sus documentos como el bolsillo que le había sido arrebatado. Esto es muy relevante, porque en el caso de las narraciones de hombres de la muestra, es común que los relatos tengan un "final feliz", es decir, ellos logran resolver los sucesos, a diferencia de las mujeres que suelen ser víctimas que sufren en este mismo tipo de historias. Al igual que en el ejemplo (1), el narrador habla de sus propias vivencias; de hecho, el antagonista de la historia pierde relevancia, cuando lo que el narrador quiere manifestar es que él logra resolver los hechos y recuperar sus pertenencias, poniendo fin a la historia. Resultados similares obtiene Soler (2004). 
Finalmente, exponemos el ejemplo (3), que si bien es representativo del cuarto tema más empleado en nuestros relatos (narraciones vinculadas con los hijos), es significativo por su uso preponderante en informantes mujeres.

(3) I: <resumen > no/ cuando pasé el susto grande fue cuando/ cuando se rompió el ojo/ con $/</$ resumen $>$ $<$ orientación $>$ estábamos en la tienda $\mathrm{Z}<$ /orientación $><$ acción de complicación $>$ y se apoyó en uno estaba detrás de la pelota ¿cachái ${ }^{12}$ ? y agarró la pelota y se paró/ <orientación> bueno tenía como un año tres meses más o menos / cuatro meses/ ni caminaba $/</$ orientación $>$ y se paró/ y tocó con esas cuestiones las vitrinas de acrílico que tienen las/ el mall13/ donde tienen puestas la zapatilla en las tiendas y chocó así/ en el ojo izquierdo de lado así el término del ojo y llegó corriendo así con las manos en la cara mamá/ mamá // E: ¿tú no te habías dado cuenta? / / I: no $\mathrm{po}^{14}$ / si yo le dije ya/ X/ mira la pelota / y yo le tiré la pelota al lugar y eso era lo más terrible porque yo me sentía súper culpable porque yo tiré la pelota y cayó justo ahí / entonces el cabro chico ${ }^{15}$ la tomó/ y hizo esto y/ y llegó llorando si no tenía sangre nada/de repente/ el V lo tomó en brazos/ qué le pasó mi niño / y ahí al costado izquierdo toda la sangre todo chorreando por la cara / y ahí no le paraba / no le paraba pero con nada/ huevón ${ }^{16} /$ pero con nada/hasta que hablamos con un con uno de los gerentes de la tienda $\mathrm{Z}$ del mall $\mathrm{X} / \mathrm{y} / \mathrm{y}$ que nos diera una solución porque no sabíamos qué hacer</acción de complicación>//E: ¿y fue como escándalo? <resolución> no/ el/ llamamos al guardia / /E: pero todos se dieron cuenta alguna gente/ pero bien piola ${ }^{17}$ fue/ le dijimos al guardia si tenía alguna enfermera/ o sea/enfermería sala de enfermería alguna enfermera en la tienda alguna cuestión po y ahí nos llevaron a la clínica/ y le pagaron todo al E porque lo tuvieron que operar del ojo / /E: ya ¿pero no le pasó nada en la vista?/ no/ si está bien

12 En español de Chile coloquial, “cachar" es "entender", "comprender", “saber", “conocer".

Centro comercial.

En español de Chile, variante de "pues".

En español de Chile, "niño".

En español de Chile, vocativo familiar.

En español de Chile, "disimulado e inadvertido". 
del ojito / pero igual le quedó la cicatriz po/ es que cada vez que el E va creciendo la cicatriz se le está corriendo/ cachái/ porque ahora la tiene por acá/ por ejemplo/ empezó ahí con la cicatriz/ ahí era/ ahí se pegó ahora la tiene por acá/ si a medida que va creciendo la cuestión chica se va corriendo va a llegar con la cicatriz por acá huevón/ en la oreja/ y sí/ esa</ resolución>. (MBIM060)

En el ejemplo (3), claramente el protagonista del relato no es la narradora, sino su hijo; de hecho, en el resumen se señala que va a contar cuando a su hijo se le rompió su ojo. Consecuentemente, es el accidente de su hijo lo que hace que su relato cobre importancia, y -a la vez- se vuelve relevante el susto que ella tuvo, pero no es lo más trascendental, porque, en efecto, no es el tema que ocupa la mayor parte de sus cláusulas narrativas, sino lo que le aconteció al niño. Las narraciones que se basan en temas que involucran hijos son muy relevantes en nuestra muestra de estudio, porque están muy asociadas a las informantes mujeres (cf. Prieto y San Martín, 2002-2003 y Coates, 2009). Como ya se señaló, entre los temas que pertenecen al dominio de lo privado ${ }^{18}$, está el que involucra hijos, por lo tanto, en este análisis hemos comprobado también este rasgo como una característica del español hablado en Santiago de Chile.

\subsubsection{Análisis de los protagonistas de las narraciones}

En lo que respecta a la presentación de un protagonista de los hechos narrados en las 192 historias que constituyen nuestra muestra y su vínculo con los factores sexo, edad y grupo socioeconómico, podemos señalar que es también el factor sexo el que presenta mayor variación. Presentamos la Tabla 9 para desarrollar las precisiones necesarias.

Tabla 9. Protagonistas de las narraciones según los factores sexo, edad y grupo socioeconómico

\begin{tabular}{l|c|c}
\multicolumn{1}{c|}{ Protagonista } & Totales & Porcentaje de Frecuencia \\
\hline Primera persona & 86 & $44,8 \%$ \\
\hline Primera y tercera persona & 87 & $45,3 \%$ \\
\hline Tercera persona & 19 & $9,9 \%$ \\
\hline Total & 192 & $100 \%$ \\
\hline
\end{tabular}

18 Hall (1995, citado en Prieto y San Martín, 2002-2003), destaca la división de los dominios público y privado: "los hombres se instalaron firmemente en el recién definido mundo de los negocios, el comercio y la política; las mujeres se instalaron en el mundo privado del hogar y la familia" (Prieto y San Martín, ibíd, p. 277). 
Lo que refleja la tabla precedente es que hay una tendencia a narrar o en primera persona $(44,8 \%)$ o incluyendo primera y tercera persona $(45,3 \%)$, pero que, en general, los relatos que solo hablan de una tercera persona con el rol protagónico son muy pocos (19/192; 9,9\%).

Respecto de lo que ocurre al desarrollar la prueba ji cuadrado que nos permite comprobar nuestra hipótesis de estudio, es decir, la correlación entre los factores externos y los internos, podemos señalar que son los hombres y las mujeres de 55 años y más del total de la muestra quienes presentan significación estadística en el empleo de primera persona como protagonista de los sucesos narrados; así, en el caso de los informantes masculinos $p=0,013$, y en las mujeres $p=0,005$. Asimismo, los hombres de 20 a 34 años también mostraron significación, donde $p=0,019$. El análisis meramente descriptivo de los datos expuestos en la tabla precedente señala que son los hombres de 55 años y más del grupo socioeconómico medio quienes tienden a narrar en primera persona, lo que no es de extrañar considerado los datos aportados por la prueba estadística.

En relación con el uso de primera y tercera persona, la prueba ji cuadrado señala que nuevamente son los hombres de 20 a 34 y los de 55 años y más de los cuatro grupos socioeconómicos en estudio quienes presentan significación estadística, ya que $\mathrm{p}=0,035$ y $\mathrm{p}=0,005$, respectivamente. Finalmente, en el empleo de terceras personas como protagonistas de los hechos, la prueba ji cuadrado señala que ninguna de las interacciones efectuadas es estadísticamente significativa.

En definitiva, hemos comprobado que son los hombres jóvenes y los del tercer grupo de edad quienes presentan menor incidencia del azar en la incorporación de primera persona, y de primera y tercera personas en sus narraciones, es decir, estos grupos de hombres se comportarían de dicha manera en la comunidad de habla en estudio y no solo en la muestra analizada.

A continuación, a través de los ejemplos (4), (5) y (6) evidenciaremos el funcionamiento de la inclusión de protagonistas en las historias.

(4) <orientación> sí // graciosa / por ejemplo/ una vez cuando yo estaba recién pololeando con tu madre que también estudiaba en la misma universidad/ iba con un maletín y con ella de un brazo $</$ orientación $><$ acción de complicación $>$ y subo una escala y me tropecé en el primer escalón y para no caerme 
fui dando saltitos hasta llegar al último escalón $</$ acción de complicación $><$ coda $>$ simpático $</$ coda $>$. (MAIIH164)

En el ejemplo que acabamos de citar, se observa cómo el narrador es, a su vez, el protagonista de la historia. Si bien, señala haber ido en compañía de su pareja, ella deja de ser relevante porque él quiere enfatizar en lo que a él le ocurrió, esto es, que se tropezó y dio saltos hasta llegar al último escalón. De hecho, no relata cómo se resolvieron los sucesos, y con eso, ella pierde absolutamente el protagonismo. Las marcas de primera persona son muy preponderantes, por ejemplo, "subo", "me tropecé", "caerme" y "fui dando"; en todos los casos, como es evidente, es el "yo" el que se pone de relieve. Es más, si elidiéramos la información referente a la pareja, la historia tendría exactamente el mismo sentido, porque no necesariamente se interpreta que al protagonista le dio vergüenza lo acontecido frente a su pareja, sino que perfectamente puede tratarse de una anécdota o de una vergüenza más general, producto del descenso no habitual de la escalera.

A continuación presentamos el ejemplo (5), representativo de la inclusión de primera y tercera persona como protagonistas de los relatos.

(5) I: <resumen> en la básica / en segundo básico / es que / son dos recuerdos porque / es como de mi vida / todos los recuerdan como mis compañeros de curso $</$ resumen $>$ $<$ orientación $>$ tenía tuvimos una profe ${ }^{19}$ de primero a cuarto básico como la típica profe que inicia a los niños y era muy mala nos trataba pero muy mal así como maltrato sicológico brígido y yo era súper floja / todo el colegio / o sea malas notas y como desordenada / desordenada sobre todo $<$ / orientación $><$ acción de complicación $>$ y estábamos como con mis amigas / la X / la Y / como ¿qué vas a ser cuando grande? / recreo / un día / segundo básico y yo eeh onda como yo quiero ser actriz yo quiero ser no sé qué no me acuerdo po y yo dije como yo quiero estudiar medicina porque mi hermano mayor quería estudiar medicina en ese tiempo y yo en realidad ni siquiera conocía las cosas / no sé por qué quería eso / / medicina / / y pasó la tía $Z$ que me odiaba y me dijo / pero 
con las notas que tení / no te alcanza ni para estud/ ni para ser ascensorista cacha la vieja mala po y yo le dije eeh / me sentí tan triste / y le dije como obligada a ser profesora básica $<$ / acción de complicación > < resolución > y me agarró como de un brazo / me llevó a inspectoría y quedé condicional en segundo básico porque la tía $\mathrm{Z}$ dijo como que yo le había faltado mucho el respeto condicional $</$ resolución $><\operatorname{cod} a>$ eso es los más que me ha pasado en el colegio parece $</ \operatorname{cod} a>$. (MAIM156)

En este ejemplo, es evidente la inclusión y la relevancia de la primera persona -narradora- y de una tercera persona en los sucesos. Este tipo de relatos es muy común en mujeres, a diferencia de aquellos que solo incluyen primera persona que son muy característicos de los hombres. En este caso, en particular, el protagonismo pertenece a quien narra y a quien se transforma en su antagonista, porque sin ella, la historia no tendría sentido. En otras palabras, en (5) no se pueden eliminar las cláusulas donde se pone de relieve al otro personaje, porque la historia deja de tener sentido. Además, es tan importante la presencia de esta tercera persona en los hechos que, incluso, tiene voz y es precisamente su intervención la que genera la forma en la que terminan los hechos.

Finalmente, exponemos el ejemplo (6), donde el narrador pierde totalmente su protagonismo para asignárselo a un tercero.

(6) E: ya / eh ¿ha pasado alguna vez un / un susto grande con la salud de alguno de sus hijos? / / I: a ver/ <vacilación/> si po / con tu papá// E: ya // I: con tu papá// E: ¿y cómo fue eso? / / I: <resumen> <orientación > tenía diecisiete años $/<$ / orientación $>/ /<$ acción de complicación $>$ cuando se me ahogó con carne $</$ resumen $>$ / entonces yo pensé que como siempre ha sido así tu sabí gracioso y chistoso yo pensé que estaba haciendo chiste / <orientación $>$ fue para una navidad $<$ / orientación > y no po estaba ahogado con carne / y cuando nos dimos cuenta nos costó un / menos mal que había una persona ahí que sabía hacer el esa cuestión de apretar lo / / E: ya// I: tomarlo de la espalda y apretarlo porque si no se me ahoga no más / / E: uh// I: ese ha sido el susto más grande que hemos tenido / / porque a / a / a partir de ese ahogamiento tuvo una co/ una terrible consecuencia porque se / se/ donde lo apretaron y le pegaron tanto / se le ganaron 
unos se le inflamaron unos ganglios en la espalda</acción de complicación> / / E: ya / / I: <resolución > y estuvo dos años en tratamiento</resolución> //E: oh/ tanto tiempo// I: sí. (BIIIM045)

En este caso se observa que la narradora le atribuye todo el protagonismo al accidente que tuvo su hijo (el padre del entrevistador) al ahogarse con un trozo de carne y las consecuencias que este suceso le trajo. Este fenómeno suele ocurrir en los relatos donde se involucran hijos, como en (3), es decir, la narradora cuenta su susto, pero este no es más importante que lo que le ocasionó dicho susto, esto es, el accidente de su hijo. Además de esto, es muy interesante la forma en como se construye la historia, porque, como es evidente, las narraciones en la "conversación" a menudo se imbrican y, por consiguiente, no presentan una estructura canónica en términos labovianos. En este caso, en concreto, los componentes de resumen, orientación y complicación figuran mezclados en las cláusulas que conforman el relato.

\section{Conclusiones}

Los principales hallazgos de este estudio se sintetizan como sigue:

Sobre la incidencia del factor sexo en la construcción de narraciones de experiencia personal: En lo que respecta a la incidencia de la variable sexo en la construcción de narraciones de experiencia personal, podemos mencionar, en primer lugar, que de los 12 ejes temáticos relevados en las 192 narraciones que constituyen nuestra muestra de investigación, no hay ningún tema que no haya sido cubierto tanto por hombres como por mujeres en alguna de las 96 narraciones analizadas. Al comparar los temas seleccionados por hombres y mujeres demostramos que los temas que ellas desarrollaron y que no fueron seleccionados por los hombres que integran la muestra, son los que suelen presentarse en la literatura como más característicos del sexo femenino, primero, aquel que trata sobre hijos y, luego, el que habla de vacaciones y aventuras (cf. Tannen, 1996; Prieto y San Martín, 2002-2003; Soler, 2004 y Coates, 2009). Asimismo, hay temas que se perfilaron como más típicos de hombres, como los hechos delictuales, donde además suelen presentarse a sí mismos como héroes.

En lo que se refiere a los protagonistas de las narraciones, se percibió que los hombres tienen una mayor tendencia a hablar de ellos mismos, 
situándose como actores principales de los acontecimientos que narran, mientras que las mujeres tienden a incluir a terceras personas con roles protagónicos.

Sobre la incidencia del factor edad en la construcción de narraciones de experiencia personal: En lo que dice relación con los temas y los protagonistas de las narraciones, no se percibió gran variedad. Sin embargo, es interesante que respecto de las temáticas abordadas, haya algunas como las vergüenzas, las travesuras o bromas, los hijos y otros recuerdos desagradables que sí parecen estar más marcadas en su uso. Así, las travesuras y bromas, por ejemplo, suelen aparecer en el grupo de edad joven de la muestra (20 a 34 años) y transcurren en el colegio o en la universidad. Asimismo, los temas asociados con otros recuerdos desagradables se ubican sobre todo en el grupo de 55 años y más. De esta forma, coincidimos con Coupland, Coupland y Giles (1991, citados por Valles, 2006: 886).

Respecto de la elección de los protagonistas de los relatos y su vínculo con el factor edad, podemos señalar que no hubo variación, por lo que concluimos que sigue siendo el factor sexo el más pertinente para realizar un análisis de este tipo.

Sobre la incidencia del factor grupo socioeconómico en la construcción de narraciones de experiencia personal: El análisis de la incidencia del factor grupo socioeconómico en el desarrollo de temas de las narraciones nos permite señalar que si bien solo en cuatro categorías hubo variabilidad en la distribución según los grupos socioeconómicos, esto es, las vergüenzas, las que involucran hijos, las que hablan de otros recuerdos desagradables y las que aluden a travesuras y bromas, es de gran relevancia que haya sido el grupo socioeconómico medio alto el único que presentó los 12 ejes temáticos en sus narraciones, porque, a diferencia de lo que hubiéramos esperado, no fueron los grupos bajos los que, por acercarse más a comunidades con oralidad primaria, los que tuvieron mayor variedad en sus temas elegidos.

Por otra parte, los hallazgos obtenidos en el análisis de los protagonistas de las narraciones solo nos permiten afirmar que el empleo de primera persona es menos característico en los extremos de la escala socioeconómica, lo que permite pensar en futuros trabajos con estos grupos como foco de investigación. 
Sobre la incidencia de los factores sexo, edad y grupo socioeconómico en interacción, en la construcción de narraciones de experiencia personal: Respecto de la interacción de variables, hemos concluido fue la variable sexo la que mostró mayor variabilidad. Por su parte, en el análisis de protagonistas de las historias, hemos comprobado que son los hombres jóvenes y los del tercer grupo de edad quienes presentan menor incidencia del azar en la incorporación de primera persona, y de primera y tercera personas en sus narraciones, es decir, estos grupos de hombres se comportarían de dicha manera en la comunidad de habla en estudio y no solo en la muestra analizada.

Otras consideraciones: En el análisis presentado hemos destacado algunos hallazgos que nos parecieron particularmente importantes, sobre todo porque se condicen con otros puestos de relieve en investigaciones desarrolladas tanto en el contexto de la lengua inglesa como en el de la lengua española. Sin embargo, como hemos manifestado en nuestro marco teórico, las investigaciones que se han centrado en el nivel discursivo de la variación son escasas. Menos frecuentes todavía son las que consideran las variables edad y grupo socioeconómico, por lo tanto, la discusión de los hallazgos se desarrolla más ampliamente en relación con la variable sexo y su posible correlación con la construcción de discurso narrativo. Si bien hemos observado que es el factor sexo el que más condiciona la variación, la relevancia de comenzar a incluir otras variables externas, así como la necesidad de comenzar a vincular lo cuantitativo del método variacionista con lo cualitativo, es fundamental. 


\section{Referencias bibliográficas}

Adam, Jean-Michel y Clara-Ubaldina Lorda. (1999). Lingüistica de los textos narrativos. Barcelona: Ariel.

Blas Arroyo, José Luis. (2005). Sociolingüística del español. Desarrollos y perspectivas en el estudio de la lengua española en contexto social. Madrid: Cátedra.

Calsamiglia, Helena y Amparo Tusón. (1999). Las cosas del decir. Manual de análisis del discurso. Barcelona: Ariel.

Camargo Fernández, Laura. (2004). La Representación del Discurso en la Narración Oral Conversacional. Estudio Sociopragmático. (Tesis doctoral.). Universidad de Alcalá; Madrid, España.

Coates, Jennifer. (2009). Mujeres, hombres y lenguaje. Un acercamiento sociolingüístico a las diferencias de género. México: Fondo de Cultura Económica.

Gajardo, Consuelo. (En preparación). La construcción discursiva de madrel trabajadora: afiliación e individuación en madres de tres niveles socioeconómicos. Tesis doctoral. Santiago de Chile: Pontificia Universidad Católica de Chile.

Guerrero, Silvana. (2011a). Diferencias de género en evaluaciones de narraciones de experiencia personales en el habla juvenil de Santiago de Chile. Una aproximación sociolingüística. Revista Signos, 44 (75), 18-32.

- (2011b). Análisis sociolingüístico de las diferencias de género en patrones narrativos de historia de experiencia personal en el habla juvenil de Santiago de Chile. Boletín de Filología, Tomo XLVI (2), 85-106.

Johnstone, Barbara. (1990). Variation in discourse: Midwestern narrative style. American Speech, 65 (3), 195-214.

. (2003). Discourse analysis and narrative. En Schiffrin, D., Tannen, D. y H. Hamilton (Eds.), The Handbook of Discourse Analysis. Oxford: Blackwell Publishing. 635-650. 
Labov, William. (1972). The transformation of experience in narrative syntax, en Language in the inner city. Studies in the Black English Vernacular (pp. 635-375). Filadelfia: University of Pennsylvania Press. . (1983 [1972]). Modelos Sociolingüísticos. Madrid: Cátedra.

Labov, William y Josua Waletzky. (1967). Narrative analysis, en Helm, Jane (Ed.), Essays on the verbal and visual arts (pp.1244). Seattle: University of Washington Press.

Lenski, Gerhard. (1954). Status crystalization: A non-vertical dimension of social status. American Sociological Review, 19, 405-413.

López Morales, Humberto. (2004). Sociolingüística. Madrid: Gredos.

Macaulay, Ronald. (2003). Discourse variation. En Chambers, J. K., Trudgill, P. y N. Schilling-Estes (Eds.), The Handbook of Language Variation and Change (pp.283-305). Oxford: Blackwell Publishing.

Moreno Fernández, Francisco. (1998). Principios de sociolingüística y sociología del lenguaje Barcelona: Ariel.

Moreno Fernández, Francisco. (2012). Sociolingüística cognitiva. Proposiciones, escolios y debates. Madrid/Frankfurt: Iberoamericana/Vervuert.

Poblete, María Teresa. (1998). Los marcadores discursivo-conversacionales de más alta frecuencia en el español de Valdivia (Chile). Estudios Filológicos, 33, 93-103.

Prieto Vera, Luis. (1995-1996). Análisis sociolingüístico del dequeísmo en el habla de Santiago de Chile. Boletín de Filología, Tomo XXXV, 379-452.

Prieto Vera, Luis y AbelardoSan Martín. (2002-2003). Diferencias de género en el empleo del discurso referido: aproximación sociolingüística y pragmático-discursiva. Boletín de Filología, Tomo XXXIX, 269-303.

Reyes, Claudia. (1996). Estrategias narrativas en la zona metropolitana de Monterrey. En Rodríguez Flores y Rodríguez Alfano (Eds.), Lenguaje y sociedad: Metodología y 
análisis aplicados a El habla de Monterrey (pp. 101120). México: Trillas.

Reyes, Claudia. (2002). Algunas diferencias en las narraciones de hombres y mujeres dentro del corpus de "El habla de Monterrey". Iztapalapa, 53, 101-115.

Rojas, Cristian, Rubio, Alejandra, San Martín, Abelardoy Silvana Guerrero. 2012. Análisis pragmático y sociolingüístico de los marcadores discursivos de reformulación en el habla de Santiago de Chile. Lenguas Modernas, 40, 103-123.

San Martín, Abelardo. (2004-2005). Igual como marcador discursivo en el habla de Santiago de Chile: función pragmáticodiscursiva y estratificación social de su empleo. Boletín de Filología, Tomo XL, 201-232.

. (2011). Los marcadores interrogativos de control de contacto en el corpus PRESEEA de Santiago de Chile. Boletín de Filología, Tomo XLVI (2), 135-166.

San Martín, Abelardo. (2013). Los reformuladores de distanciamiento en el corpus PRESEEA de Santiago de Chile. Boletín de Filología, Tomo XLVIII (1), 171-199.

San Martín, Abelardo y Silvana Guerrero. (2013). Una aproximación sociolingüística al empleo del discurso referido en el corpus PRESEEA de Santiago de Chile. Revista Signos, 46, (82), 258-282.

Sharim, Dariela. (2005). La identidad de género en tiempos de cambio: Una aproximación desde los relatos de vida. Psykhe, 14 (2), 19-32.

Shiro, Martha. (2003). Genre and Evaluation in Narrative Development. Journal of Child Language, 30, 165-195.

Silva-Corvalán, Carmen. (1987). La narración oral española: estructura y significado. En Bernárdez, E. (Comp.). Lingüística del Texto. Madrid: Arco Libros.

- (2001). Sociolingüística y pragmática del español. Washington: Georgetown University Press. 
Soler, Sandra. (2004). Discurso y género en historias de vida. Una investigación de relatos de hombres y mujeres en Bogotá. Bogotá: Publicaciones del Instituto Caro y Cuervo.

Tannen, Deborah. (1990a). La comunicación entre hombres y mujeres a la hora del trabajo. Bueno Aires-Argentina: Javier Vergara Editor S. A. . (1990b). You just don't understand. Women and man in conversation. Nueva York: Ballantine Books.

Tannen, Deborah. (1996). Género y discurso. Barcelona: Paidós.

Valles, Beatriz. (2006). La cohesión y la coherencia en la conversación del paciente con demencia: un estudio discursivo. Revista de la Asociación colombiana de gerontología y geriatría, 20 (1), 881-887. 